Z Herz- Thorax- Gefäßchir 2021 · 35:317-319 https://doi.org/10.1007/s00398-021-00472-9 Angenommen: 5. November 2021

(c) The Author(s), under exclusive licence to Springer Medizin Verlag GmbH, ein Teil von Springer Nature 2021

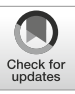

\section{Autor}

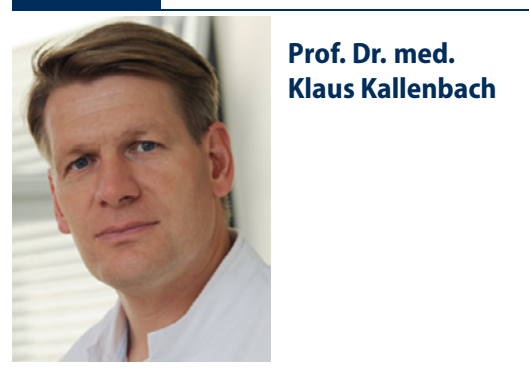

In der vorliegenden Arbeit wird aus Gründen der besseren Lesbarkeit ausschließlich die männliche Form verwendet. Sie bezieht sich auf Personen jedes Geschlechts.

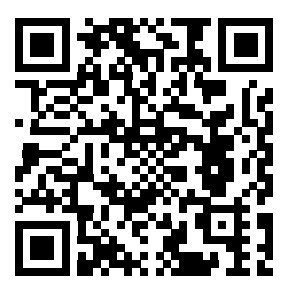

QR-Code scannen \& Beitrag online lesen

\title{
Eine kontroverse Debatte: biologischer vs. mechanischer Klappenersatz
}

\author{
Klaus Kallenbach \\ INCCI HaerzZenter, Luxembourg, Luxemburg
}

Liebe Leserinnen und liebe Leser,

in der aktuellen Ausgabe der Zeitschrift für Herz-, Thorax- und Gefäßchirurgie publizieren wir einen Beitrag von Herrn Dr. Ralf-Uwe Kühnel aus Bernau, der in der Rubrik „Im Brennpunkt" eine aktuelle Arbeit von Malvindi et al. zusammenfasst und kommentiert: „Aortic valve replacement with biological prosthesis in patients aged 50-69 years" [1]. Seine Konklusion: „Der biologische Aortenklappenersatz ist eine praktikable Option in der intermediären Altersgruppe 50 bis 70 Jahre".

Diese Aussage provoziert meinen Widerspruch, denn ich halte diese These für wissenschaftlich nicht haltbar!

Schaut man sich diese monozentrische, retrospektive Arbeit von Malvindi et al. genauer an, werden nur 132 Patienten nach biologischem Aortenklappenersatz (AKE) in der Altersgruppe 50 bis 59 Jahre über einen Zeitraum von 15 Jahren nachverfolgt. Es findet sich kein signifikanter Unterschied bezüglich der Mortalität im Vergleich zum mechanischen Aortenklappenersatz. Daraus wird geschlussfolgert, dass der biologische Klappenersatz in dieser Altersgruppe eine praktikable Option darstellt. Auch wird argumentiert, dass eine degenerierte Aortenklappe sowohl chirurgisch als auch interventionell (TAVI, ,valvein-valve ${ }^{\prime \prime}$ ) sicher ersetzt werden kann.

Schon in der Übersichtsarbeit von Head et al. [2] wird deutlich darauf hingewiesen, dass die meisten monozentrischen Studien zur Beantwortung dieser relevanten Frage schlichtweg "underpowert" sind, um eine wissenschaftlich belastbare Aussage vornehmen zu können. Zur gleichen Vermutung kommen Goldstone et al. aus Stanford [3], die immerhin 25.445 Patienten nach
Klappenersatz untersuchten. Diese Limitation der zu geringen Fallzahl trifft auch auf die Arbeit von Malvindi et al. zu. Also muss man sich besser geeigneter Studien wie RCT, "Propensity-score-matched"-Studien und Metaanalysen zuwenden:

- Ad 1.: Die 2 ursprünglichen RCT, nämlich VA- und Edingburgh-Trail $[4,5]$ zeigten ein besseres (VA) bzw. vergleichbares Überleben nach mechanischem AKE. Reoperationen waren häufiger, Blutungen seltener in den biologischen Gruppen, während Schlaganfall und Endokarditis ähnlich verteilt waren. In der aktuelleren Studie von Stassano et al. [6] von 2009 mit 310 randomisierten Patienten und einem mittleren Alter von 64 Jahren fand sich kein signifikanter Unterschied im Überleben nach 9 Jahren mittlerer Nachverfolgung. Klappenversagen und Reoperationen waren hoch signifikant häufiger nach Bio-AKE, aber es fand sich erstaunlicherweise kein signifikanter Unterschied hinsichtlich Blutungen. Auch hier kann man eine zu kleine Fallzahl und eine zu kurze Nachverfolgung vermuten.

- Ad 2: Studien, die Propensity matching oder multivariable Analysen nutzen, kommen zu unterschiedlichen Ergebnissen. Die größte bisher durchgeführte Propensity-score-Studie mit fast 40.000 älteren Patienten aus der STS-Database ([7]; Brennan et al.) zeigt einen leichten, aber signifikanten Überlebensvorteil nach mechanischem Klappenersatz. Eines aber lässt sich sicher erkennen: Es besteht ein deutlicher Zusammenhang zwischen der Mortalität und dem Implantationsalter beim biologischen AKE. 
- Ad 3.: Im Rahmen des Jahrestreffens der EACTS 2021 haben Leviner et al. [8] eine "hazard ratio meta-analysis" vorgestellt, welche den biologischen vs. mechanischen AKE vergleicht. Nach systematischer Suche der bekannten wissenschaftlichen Portale wurden 15 Studien mit 16.876 Patienten identifiziert. Die Einschlusskriterien waren rigoros: nur RCT, Propensity-scorematched- und "Inverse-probabilityweighting"-Studien wurden inkludiert. Nach mechanischem AKE waren die Mortalität und die Reoperationen niedriger, die Blutungen erhöht und die Schlaganfallrate vergleichbar. Die Autoren zeigten einen Überlebensvorteil nach mechanischem AKE bei Patienten unter 70 Jahren.

Schon diese Fakten widersprechen der Aussage, dass der biologische Aortenklappenersatz im Alter zwischen 50 und 70 Jahren eine sichere Option ist. Zahlreiche Studien zeigen ein besseres Überleben nach mechanischem, aber keine einzige nach biologischem Aortenklappenersatz. Ein weiteres, oft unbenanntes Problem: Im Rahmen des EACTS Annual Meeting 2021 haben gleich mehrere TAVI-Kardiologen (sic!) davor gewarnt, dass der Zugang zu den Koronarostien nach einer Valve-invalve-TAVI oft nicht mehr möglich ist. Wollen Sie einem 50-jährigen Patienten eine biologische Klappenprothese einsetzen, die wahrscheinlich innerhalb der ersten 10 Jahre degeneriert, und ihm dann im Alter von 60 per TAVI valve-in-valve jede interventionelle Therapie einer möglichen KHK verwehren? Klingt nach keiner guten Idee...

\section{\) Zahlreiche Studien zeigen ein besseres Langzeitüber- leben nach mechanischem Aortenklappenersatz}

Ein weiteres Argument für den mechanischen AKE bei jüngeren Patienten: Die FDA in den USA hat die OnX-AortenklappenProthese für eine INR von 1,5-2 zugelassen. Derzeit laufen Studien, ob eine Antikoagulation mit Apixaban statt mit Vitamin-KAntagonisten möglich ist. Damit wäre das stärkste Argument für den biologischen AKE obsolet.
Verstehen Sie mich bitte nicht falsch: Der biologische AKE hat seinen Stellenwert. Aber ich warne, auch hinsichtlich der Tendenz, die Altersgrenze für TAVI nach unten zu verschieben, davor, die Risiken des biologischen AKE bei jüngeren Patienten zu ignorieren.

In der Hoffnung, diese wichtige Diskussion weiter belebt zu haben, wünsche ich Ihnen viel Spaß beim Lesen der Zeitschrift für Herz-, Thorax- und Gefäßchirurgie!

Herzlichst
Ihr
Klaus Kallenbach

\section{Korrespondenzadresse}

Prof. Dr. med. Klaus Kallenbach

INCCI HaerzZenter

$2 A$ rue N.E. Barblé, 1210 Luxembourg,

Luxemburg

kallenbach.klaus@incci.lu

Interessenkonflikt. K. Kallenbach gibt an, dass kein Interessenkonflikt besteht.

\section{Literatur}

1. Malvini PG et al (2021) Aortic valve replacement with biological prosthesis in patients aged 50-69 years. Eur J Cardiothorac Surg 59(5):1077-1086

2. Head SJ et al (2017) Mechanical versus bioprosthetic aortic valve replacement. Eur Heart J 38:2183-2191

3. Goldstone AB et al (2017) Mechanical or Biologic Prostheses for Aortic-Valve and Mitral-Valve Replacement. NEng J Med 377(19):1847-1857

4. Hammermeister K et al (2000) Outcomes 15 years after valve replacement with a mechanical versus a bioprosthetic valve: final report of the Veterans Affairs randomized trial. J Am Coll Cardiol 36:1152-1158

5. Oxenham Hetal (2003) Twenty year comparison of a Bjork-Shiley mechanical heart valve with porcine bioprostheses. Heart 89:715-721

6. Stassano P et al (2009) Aortic valve replacement: a prospective randomized evaluation of mechanical versus biological valves in patients aged 55 to 70 years. J Am Coll Cardiol 54:1862-1868

7. Brennan JM et al (2013) Long-term safety and effectiveness of mechanical versus biologic aortic valve protheses in older patients: results from the Society of Thoracic Surgeons Adult Cardiac Surgery National Database. Circ 127(16):1647-55

8. Leviner D et al. Mechanical vs. bioprosthetic aortic valve replacement in patients younger than 70 years of age - a hazard ratio meta-analysis. EACTS Daily News, 13. Okt. 2021

\section{Springer Medizin Podcast} Medizin für Gesundheitsprofis

Der Podcast von SpringerMedizin.de geht seit Juli 2020 spannenden Fragen aus der Welt der Medizin nach immer freitags erweitert eine neue Folge das bereits bestehende OnlineAngebot.

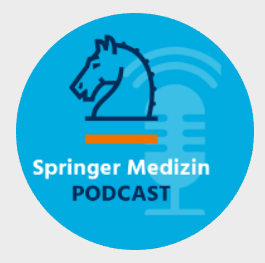

„Mit unserem Podcast möchten wir all jene ansprechen, die sich auf medizinische Themen in einer, gewissen

Flughöhe' einlassen möchten“, erklärt Dr. Erik Heintz, Chefredakteur von SpringerMedizin.de das neue Format. "Gemeint sind damit Menschen, die sich nicht mit medizinischem Halbwissen zufrieden geben und gerne mehr wissen möchten. Daher legen unsere Redakteurinnen und Redakteure in München und Heidelberg vor allem auf den inhaltlichen Anspruch großen Wert. Unter Bezugnahme auf neue Studien, neue Erkenntnisse und praxisrelevantes Wissen bereiten wir jeden Podcast gründlich vor. Die Themen drehen sich um Gesundheit, Krankheit, Diagnostik, Therapie und Prävention, zu denen wir unsere Fachexpertinnen und Fachexperten aus verschiedenen medizinischen Fachgebieten hinzuziehen."

So entstehen abwechslungsreiche Folgen, die sowohl detailliert Leitlinien abarbeiten, der Evidenz von neuen Studienergebnissen auf den Grund gehen, aber auch die Hintergründe zu Erkrankungen und Therapien aufschlüsseln - sodass einerseits die ärztliche Praxis erleichtert und konkrete Tipps für die alltägliche Arbeit vermittelt werden, andererseits aber auch der Horizont über das eigene Fachgebiet hinaus erweitert werden kann.

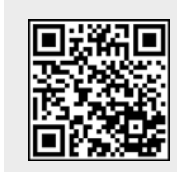

www.springermedizin.de/podcast 
Hier steht eine Anzeige.

黑 Springer 